DOI: 10.5582/ddt.2021.E1

\title{
Retracted: The safety of ritodrine hydrochloride: Adverse effects on fetuses and newborns
}

This article entitled "The safety of ritodrine hydrochloride: Adverse effects on fetuses and newborns" (1) has been retracted at the request of the authors due to wrong data extraction and processing.

\section{Reference}

1. Yonaga Y, Ito A. The safety of ritodrine hydrochloride: Adverse effects on fetuses and newborns. Drug Discov Ther. 2021; 15(1):14-19. DOI: 10.5582/ddt.2021.01016. 


\title{
The safety of ritodrine hydrochloride: Adverse effects on fetuses and newborns
}

\author{
Yuriko Yonaga*, Akihiko Ito \\ Department of Pharmaceutical Sciences, Teikyo Heisei University, Nakano, Tokyo, Japan.
}

\begin{abstract}
SUMMARY Prematurely born infants face unique risks, and the treatment of imminent preterm birth is thus an important part of perinatal care. Ritodrine hydrochloride (Rito) is widely used as a therapeutic agent to treat imminent preterm birth in Japan. Following assessment of the risks and benefits of shortacting $\beta$-agonists, including Rito, in Europe, however, the use of Rito has begun to be questioned. Thus, in this study we investigated the safety of Rito in the treatment of imminent preterm birth, with a particular focus on the adverse effects (AEs) on fetuses and newborn infants. Using the Pharmaceuticals and Medical Devices Agency of Japan's Japanese Adverse Drug Event Report (JADER) database, the AEs on fetuses and newborns caused by oral and injected Rito were extracted and analyzed. The reported odds ratios for oral Rito were significantly higher for fetal tachycardia, fetal bradycardia, neonatal hypoglycemia, and neonatal heart failure than for other drugs. The reported odds ratios for Rito injection were significantly higher for fetal tachycardia and neonatal hypoglycemia than for other drugs. Oral drugs had more adverse effect reports than injectable drugs.
\end{abstract}

Keywords Ritodrine hydrochloride, pregnant women, fetuses, newborns, adverse effects

\section{Introduction}

Perinatal care strives to protect the health of fetuses and newborn infants. In recent years, the average age of first marriage for women has increased, and late marriage is becoming more frequent. With the increase in late marriage, the average maternal age is also on the rise (1). The Japan Society of Obstetrics and Gynecology (JSOG) defines advanced maternal age as primiparas more than 35 years of age. Advanced maternal age can increase the risk of preterm labor as a result of aged ova and increased chromosomal abnormalities of the fertilized egg. The JSOG defines imminent preterm birth as when regular uterine contractions are observed between 22 and 36 weeks of gestation and progress in the degree of cervical dilatation/extension is observed, or when the cervical canal is examined at the first visit and is dilated by $2 \mathrm{~cm}$ or more (2). Infants born prematurely face a variety of risks and challenges. Preterm birth in late pregnancy (34-36 weeks gestation) can result in respiratory problems, hypothermia, hypoglycemia, etc. Preterm birth at an earlier stage (less than 34 weeks gestation) poses risks such as severe respiratory distress, cerebral hemorrhage, and severe infections $(3,4)$. Therefore, the treatment of preterm labor in perinatal medicine is important.

Rito and magnesium sulfate are covered by insurance in Japan as tocolytic agents used in the treatment of imminent preterm birth, and Rito is widely used $(5,6)$. Drug selection and dose adjustment generally take into consideration the subjective symptoms of uterine contraction and length of the cervical canal. Rito has a selective $\beta 2$ receptor-stimulating effect and is used in pregnant women after 16 weeks of gestation. Depending on the patient's symptoms, continuous infusion for $24 \mathrm{~h}$ by oral administration or infusion is performed. Although it has been reported that the administration of Rito prolongs the gestation period and increases the rate of term delivery $(7,8)$, its use has begun to be questioned $(9,10)$.

Currently, the use of Rito to treat preterm labor varies greatly from country to country. In October 2013, a risk/benefit assessment of short-acting $\beta$-agonists, including Rito, was conducted in Europe. For oral preparations, the cardiovascular risk was judged to outweigh the efficacy and approval was revoked (11). The use of injections was limited to a maximum of 48 $\mathrm{h}$ between the $22 \mathrm{nd}$ and $37 \mathrm{th}$ week of gestation due to concerns related to cardiovascular risk (12). At that time, there were few research reports recommending against long-term administration in Japan. Since longterm administration for the purpose of continuing pregnancy is a widely used treatment method, administration with careful monitoring for adverse 
effects (AEs) has been considered a viable option.

Recently, however, there have been reports that question the efficacy of Rito and query if it should be withheld due to concerns related to serious $\operatorname{AEs}(9,10)$. Although pregnant women may need this medication, information related to its administration during pregnancy remains unclear and there is little information in the package insert. Against this background, there are many concerns about the effects on fetuses and newborns. Therefore, in this study, we analyzed the AEs on fetuses and newborns to determine the safety of Rito in the treatment of imminent preterm birth.

\section{Methods}

\subsection{Usage survey of Rito usage}

Using the Ministry of Health, Labour and Welfare's NDB Open Data, the amount of Rito (oral medicine and injectable) used from April 2014 to March 2018 was investigated.

2.2. Extraction of AEs on fetuses and neonates caused by Rito (oral medicine and injectable)

Using the Pharmaceuticals and Medical Devices Agency of Japan's Japanese Adverse Drug Event Report (JADER) database, we analyzed the adverse effect data reported for approximately 15 years (April 2004-December 2019), and extracted the AEs caused by Rito. Then, AEs related to fetuses and newborn infants counted. Further, the number of adverse effect reports for drugs other than Rito was counted for the AEs related to fetuses and newborn infants listed in Rito.

\subsection{Analysis of AEs reporting frequency}

To understand the frequency of AEs related to fetuses and newborn infants caused by Rito, a $2 \times 2$ contingency table was created for each adverse effect according to the presence or absence of Rito administration and the presence or absence of AEs. Then, the reported odds ratio (ROR) and the $95 \%$ confidence interval were calculated. For drugs other than Rito, the keywords "fetus," "newborn," "pregnancy," and "caesarean section" were used, and only cases in which it was clear that the patient was pregnant were extracted.

2.4. Comparison of AEs tendency between oral and injectable Rito

To compare the frequency of reports of AEs common to oral Rito and injectable drugs, a $2 \times 2$ contingency table was prepared according to administration method (oral administration or injection) and presence or absence of AEs. The ROR and $p$-value were calculated. The $\operatorname{lnROR}$ and $-\log \mathrm{P}$ values were also graphed, and the frequency of reported adverse reactions with oral and injectable drugs was compared and examined.

\subsection{Statistical analysis}

Fisher's exact test was used to compare the two groups, and the level of significance was set at $p<0.05$.

\section{Results}

\subsection{Oral Rito}

\subsubsection{Usage status of oral Rito}

Figure 1 shows the usage status of oral Rito by year and age. The amount of oral Rito used in FY2014 was smaller than in other years, but it was almost the same in FY2015-2017. In addition, when compared by age, the usage was highest in patients 30 to 34 years old, followed by 25 to 29 years old and 35 to 39 years old in all years. Although the amount used was smaller than that of the above age groups, it was also used in patients in their 40s and older, early 20s, and 15 to 19 years old.

3.1.2. Reporting frequency of AEs on fetuses and newborns

The total number of reported adverse reactions caused by oral Rito was 260 . Adverse reaction in fetuses was 17 , and in newborns was 33 .

In fetuses, the most frequently reported adverse drug reaction was fetal tachycardia (5 cases, 29.4\%). This was followed by fetal bradycardia (4 cases, $23.5 \%$ ), fetal disorder ( 2 cases, $11.8 \%$ ), and fetal death ( 2 cases, $11.8 \%$ ). In addition, in order to examine the frequency of the reported AEs of Rito, we compared it with other drugs. As a result, the RORs for fetal tachycardia and fetal bradycardia were 9.468 (2.984-30.042) and 3.292 (1.093-9.920), respectively. The frequency of reported adverse reactions to Rito was significantly higher than

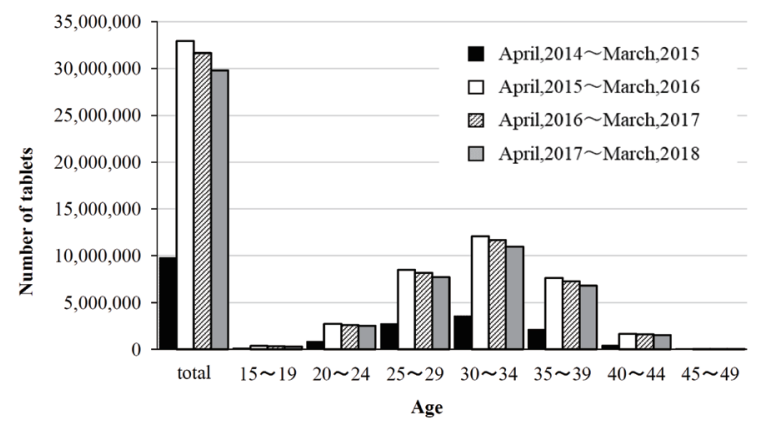

Figure 1. Annual usage of ritodrine hydrochloride (5 mg tablet). Using the Ministry of Health, Labour and Welfare's NDB Open Data, the amount of ritodrine hydrochloride (oral medicine) used from April 2014 to March 2018 was investigated. 
Table 1. Adverse effects and reported odds ratios for oral ritodrine hydrochloride: Fetuses

\begin{tabular}{|c|c|c|c|c|}
\hline \multirow{3}{*}{ Adverse effects } & \multicolumn{2}{|c|}{ Number of reports } & \multirow{3}{*}{ ROR $(95 \%$ CI) } & \multirow{3}{*}{$p$ value } \\
\hline & Rito & Other drugs & & \\
\hline & Total: 260 & Total: 3387 & & \\
\hline Fetal tachycardia & 5 & 7 & $9.468(2.984-30.042)$ & 0.0000 \\
\hline Fetal bradycardia & 4 & 16 & $3.292(1.093-9.920)$ & 0.0249 \\
\hline Fetal disorder & 2 & 17 & $1.537(0.353-6.688)$ & 0.5639 \\
\hline Fetal death & 2 & 91 & $0.281(0.069-1.146)$ & 0.0587 \\
\hline
\end{tabular}

The data shows the top four adverse effects. Rito: ritodrine hydrochloride, ROR: reported odds ratio, CI: confidence interval.

Table 2. Adverse effects and reported odds ratios for oral ritodrine hydrochloride: Newborns

\begin{tabular}{|c|c|c|c|c|}
\hline \multirow{3}{*}{ Adverse effects } & \multicolumn{2}{|c|}{ Number of reports } & \multirow{3}{*}{ ROR $(95 \% \mathrm{CI})$} & \multirow{3}{*}{$p$ value } \\
\hline & Rito & Other drugs & & \\
\hline & Total: 260 & Total: 3387 & & \\
\hline Neonatal hypoglycemia & 8 & 31 & $3.437(1.563-7.555)$ & 0.0011 \\
\hline Low birth weight infants & 8 & 320 & $0.304(0.149-0.621)$ & 0.0005 \\
\hline Neonatal heart failure & 5 & 10 & $6.622(2.246-19.519)$ & 0.0000 \\
\hline Neonatal asphyxia & 3 & 187 & $0.200(0.063-0.629)$ & 0.0023 \\
\hline
\end{tabular}

The data shows the top four adverse effects. Rito: ritodrine hydrochloride, ROR: reported odds ratio, CI: confidence interval.

that of other drugs. The RORs for fetal disorder and fetal death were 1.537 and 0.281 , respectively, but no significant difference was observed (Table 1).

The most frequently reported adverse drug reaction in newborns was neonatal hypoglycemia ( 8 cases, $24.2 \%$ ), followed by low birth weight infant ( 8 cases, $24.2 \%$ ), neonatal heart failure ( 5 cases, $15.2 \%$ ), and neonatal asphyxia (3 cases, $9.1 \%$ ). As a result of comparison with other drugs, the ROR of neonatal hypoglycemia was 3.437 (1.563-7.555), and the ROR of neonatal heart failure was 6.622 (2.246-19.519). This result indicates that the frequency of reported adverse reactions due to Rito is significantly higher than that of other drugs. In contrast, the ROR for low birth weight infants was $0.304(0.149-0.621)$ and the ROR for neonatal asphyxia was $0.200(0.063-0.629)$. The frequency of these AEs due to Rito was significantly lower than that of other drugs (Table 2).

\subsection{Rito injection}

\subsubsection{Usage status of Rito injection}

Figure 2 shows the amount of Rito injections by year and age. The total amount of Rito injections was approximately the same in the four years from April 2014 to March 2018. By age, the usage was highest in patients 30 to 34 years old, followed by approximately the same usage in patients 25 to 29 years old and 35 to 39 years old. Although the amount used was smaller than that of the above age groups, it was also used in patients in their 40s and older, early 20s, and 15 to 19 years old.

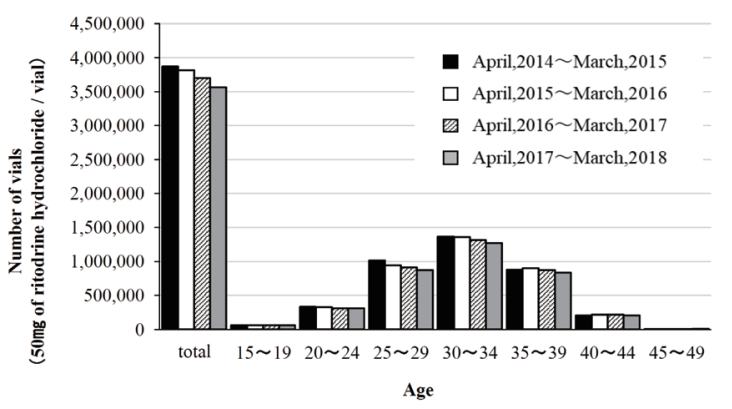

Figure 2. Annual usage of ritodrine hydrochloride injection. Using the Ministry of Health, Labour and Welfare's NDB Open Data, the amount of ritodrine hydrochloride (injectable) used from April 2014 to March 2018 was investigated.

3.2.2. Reporting frequency of AEs on fetuses and newborns

The total number of reported adverse reactions caused by Rito injection was 1281 . Adverse reaction in fetuses was 48 , and in newborns was 94 .

The most frequently reported adverse reactions in fetuses were fetal tachycardia (10 cases, $20.8 \%$ ), followed by fetal death ( 8 cases, $16.7 \%$ ), transient fetal bradycardia abnormality ( 6 cases, $12.5 \%$ ), and fetal bradycardia (5 cases, 10.4\%). In comparison with other drugs, fetal tachycardia was significantly higher (ROR 3.556 [0.976-12.951]). In the following three items, the reported adverse effect rate of Rito was significantly lower than that of other drugs: fetal death (ROR 0.149 [0.071-0.314]), fetal transient bradycardia abnormality 
Table 3. Adverse effects and reported odds ratios for ritodrine hydrochloride injection: Fetuses

\begin{tabular}{|c|c|c|c|c|}
\hline \multirow{3}{*}{ Adverse effects } & \multicolumn{2}{|c|}{ Number of reports } & \multirow{3}{*}{$\operatorname{ROR}(95 \% \mathrm{CI})$} & \multirow{3}{*}{$p$ value } \\
\hline & Rito & Other drugs & & \\
\hline & Total: 1281 & Total: 1359 & & \\
\hline Fetal tachycardia & 10 & 3 & $3.556(0.976-12.951)$ & 0.0400 \\
\hline Fetal death & 8 & 55 & $0.149(0.071-0.314)$ & 0.0000 \\
\hline Fetal transient bradycardia abnormalities & 6 & 39 & $0.159(0.067-0.378)$ & 0.0000 \\
\hline Fetal bradycardia & 5 & 44 & $0.117(0.046-0.296)$ & 0.0000 \\
\hline
\end{tabular}

The data shows the top four adverse effects. Rito: ritodrine hydrochloride, ROR: reported odds ratio, CI: confidence interval.

Table 4. Adverse effects and reported odds ratios for ritodrine hydrochloride injection: Newborns

\begin{tabular}{|c|c|c|c|c|}
\hline \multirow{3}{*}{ Adverse effects } & \multicolumn{2}{|c|}{ Number of reports } & \multirow{3}{*}{ ROR $(95 \% \mathrm{CI})$} & \multirow{3}{*}{$p$ value } \\
\hline & Rito & Other drugs & & \\
\hline & Total: 1281 & Total: 1359 & & \\
\hline Neonatal hypoglycemia & 23 & 21 & $1.165(0.642-2.115)$ & 0.6157 \\
\hline Low birth weight infant & 21 & 86 & $0.247(0.152-0.400)$ & 0.0000 \\
\hline Preterm infant & 9 & 87 & $0.103(0.052-0.206)$ & 0.0000 \\
\hline Neonatal asphyxia & 8 & 126 & $0.061(0.030-0.126)$ & 0.0000 \\
\hline
\end{tabular}

The data shows the top four adverse effects. Rito: ritodrine hydrochloride, ROR: reported odds ratio, CI: confidence interval.

(ROR 0.159 [0.067-0.378]), and fetal bradycardia (ROR 0.117 [0.046-0.296]) (Table 3).

In newborns, the most frequently reported adverse drug reaction was neonatal hypoglycemia (23 cases, $24.5 \%)$; this was followed by low birth weight infant ( 21 cases, $22.3 \%$ ), preterm infant (9 cases, $9.6 \%$ ), and neonatal asphyxia (8 cases, $8.5 \%$ ). As a result of comparison with other drugs, the reported adverse effect rate of Rito for low birth weight infant, preterm infant, and neonatal asphyxia was significantly lower than that of other drugs (Table 4).

3.3. Comparison of adverse effect tendency between oral and injectable Rito

AEs common to oral and injectable drugs include fetal tachycardia, fetal bradycardia, fetal death, neonatal hypoglycemia, low birth weight infant, and neonatal asphyxia. The relationship between $\operatorname{lnROR}$ and $-\log \mathrm{P}$ reveals that AEs tended to occur more easily with oral medication for all six reported adverse drug reactions (Figure 3). The order of frequency of occurrence was fetal bradycardia, fetal tachycardia, low birth weight infant, neonatal asphyxia, neonatal hypoglycemia, and fetal death. For fetal bradycardia, the frequency of AEs was significantly higher.

\section{Discussion}

A survey of Rito usage revealed low usage in 2014. This was likely influenced by the October 2013 report on the

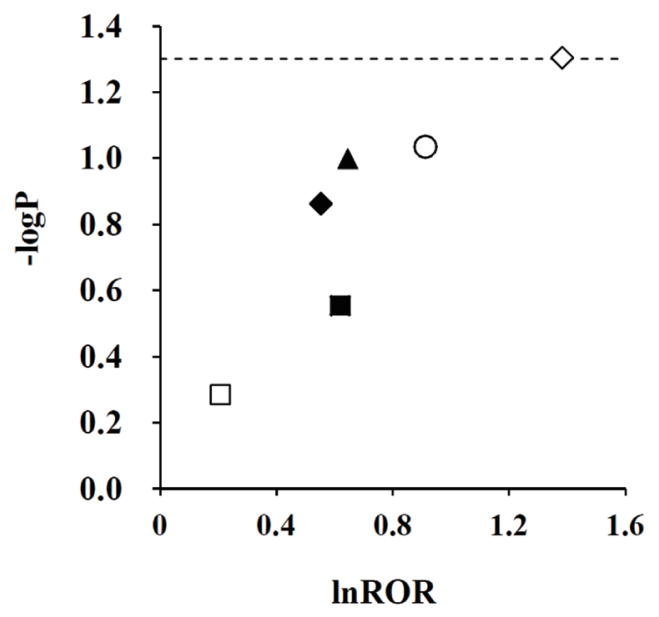

Figure 3. Comparison of the frequency of adverse effects common to oral ritodrine hydrochloride and injectable drugs. Fetal tachycardia (०), Fetal bradycardia $(\diamond)$, Fetal death $(\square)$, Neonatal hypoglycemia $(\bullet)$, Low birth weight infants $(\boldsymbol{\Delta})$, Neonatal asphyxia (๘), dotted line: $-\log 0.05$. $\ln R \mathrm{OR}$ and $-\log \mathrm{P}$ values were graphed, and the frequency of reported adverse reactions with oral and injectable drugs was compared and examined. The positive direction on the horizontal axis tends to be more likely to occur due to oral administration, and the positive direction on the vertical axis indicates that there is a statistically significant difference.

domestic response to the restriction on the use of shortacting $\beta$-agonists, including Rito, in Europe. In all of the years surveyed, both oral and injectable drugs were used most frequently in patients between 30 and 34 years old, followed by those aged 25 to 29 and 35 to 39 years old. Although the amount used was smaller than in that 
of the above age groups, Rito was also used in patients in their 40s and older, early 20s, and 15 to 19 years old. According to the data from the "Annual transition of the number of births by mother's age (5-year-old class) and birth order (2015-2018)" by the Ministry of Health, Labour and Welfare, the number of births was the highest at 30 to 34 years old, followed by 25 to 29 years old and 35 to 39 years old, indicating that usage was linked to birth status. Rito was also used by patients in their 40s and older. This may be due to the fact that as maternal age increases as a result of later marriage, and as the risk of imminent premature birth increases with age, the use of this medication becomes increasingly necessary.

Against this background, we analyzed the status of AEs reported in fetuses and newborns caused by oral and injectable Rito. We found that the RORs of oral Rito were significantly higher for fetal tachycardia, fetal bradycardia, neonatal hypoglycemia, and neonatal heart failure than for other drugs. The RORs for Rito injection were significantly higher for fetal tachycardia and neonatal hypoglycemia than for other drugs. They were therefore considered to be AEs unique to Rito. These adverse effect reports were consistent with the already reported package inserts $(12,13)$.

Rito is more selective for uterine muscle than other $\beta$-agonists, such as isoxsuprine hydrochloride and isoproterenol hydrochloride. Many AEs, including fetal tachycardia and fetal bradycardia, have been reported due to the pharmacological action of stimulating $\beta$-receptors, however. Rito's longer period of action can lead to the postnatal effect of neonatal heart failure in infants. In addition, Rito has hepatic glycogenolysis and insulin secretion suppression effects, which are related to the increased maternal blood glucose effect. It is known that the rebound can cause transient hypoglycemia in postnatal infants $(14,15)$. Hyperglycemia and diabetic ketoacidosis have been reported as serious adverse reactions to Rito in the mother. The onset of neonatal hypoglycemia can be suppressed by controlling the blood glucose at an early stage of the onset of maternal AEs.

The reporting frequency of the common AEs, such as fetal tachycardia, fetal bradycardia, fetal death, neonatal hypoglycemia, low birth weight infant, and neonatal asphyxia, between oral and injectable Rito was compared. All six AEs tended to occur more easily with oral medicine than with injectable drugs. The treatment of imminent preterm birth in Japan may be related to the long-term use of oral medication. Although it is not possible to directly compare the number of people in the usage survey, it is presumed that there is a large difference in usage and that oral medications are often used. In addition, reports from pharmaceutical companies also indicate that oral medications are often used in Japan.

As for the domestic administration status of Rito preparations (April 2012-March 2013), of the 2688 treatment groups, 2148 (79.9\%) were treated with Rito alone, and $113(4.2 \%)$ were treated with intravenous drip alone. Both oral and infusion treatments were reported in 427 patients $(15.9 \%)(11)$. According to the pharmaceutical Interview Form, the number of AEs per daily average dose increased as the doses of both oral and injectable drugs increased (oral medicine: $11-15 \mathrm{mg}$ [2.2\%], $16 \mathrm{mg}$ [4.1\%]; injectable medicine: 151-200 $\mu \mathrm{g}$ [17.5\%], $201 \mu \mathrm{g}[31 \%])(16,17)$. It is known that the number of adverse drug reactions for each period of use is highest 3 days after the start of administration (oral medicine $1.3 \%$; injection $14.0 \%$ ), however, and does not change significantly, even if the period of use is extended $(12,13)$.

All six reported adverse drug reactions in fetuses and newborn infants investigated herein were found to be more likely to occur with oral drugs than with injectable drugs. In fetuses and newborns, long-term administration of this drug may increase the risk of AEs compared to short-term administration. In Europe and the United States, short-term administration within $48 \mathrm{~h}$ by injectable drug is recommended. The results herein indicate that the treatment period should be as short as possible.

Funding: None.

Conflict of Interest: The authors have no conflicts of interest to disclose.

\section{References}

1. Ministry of Health, Labour and Welfare of Japan. Demographic statistics 2018. https://www.mhlw.go.jp/ toukei/saikin/hw/jinkou/geppo/nengail8/index.html (accessed January 14, 2021). (in Japanese)

2. Japan Society Obstetrics and Gynecology. Guideline for Gynecological Practice in Japan 2017, Tokyo. (in Japanese)

3. Mathews TJ, Driscoll AK. Trends in Infant Mortality in the United States, 2005-2014. NCHS Data Brief. 2017; 279:1-8.

4. MacDorman MF, Mathews TJ, Mohangoo AD, Zeitiln J. International comparisons of infant mortality and related factors. United States and Europe, 2010. Natl Vital Stat Rep. 2014; 63:1-6.

5. Takagi K, Satoh K, Multicentre Premature Labour Study G: Is long-term tocolysis effective for threatened premature labour? J Int Med Res. 2009; 37:227-239.

6. de Heus R, Mol BW, Erwich JJ, Van Geijn HP, Gyselaers WJ, Hanssens M, Harmark L, Van Holsbeke CD, Duvekot J, Schobben FFAM, Wolf H, Visser GHA. Adverse drug reactions to tocolytic treatment for preterm labour: prospective cohort study. BMJ. 2009; 338:b744.

7. Suemitsu A, Suzuki M, Suzuki M, Funakoshi R. The safety using ritodrine for long-term tocolysis - The effects of the provision of drug information by pharmacists. Jpn J Drug Inform. 2019; 21:104-108. (in Japanese)

8. Neilson JP, West HM, Dowswell T. Betamimetics for inhibiting preterm labour. Cochrane Database Syst Rev. 
2014; 2:CD004352.

9. Nakamura M, Hasegawa J, Arakaki T, Hamada S, Takita H, Oba T, Koide K, Matsuoka R, Sekizawa A. Comparison of perinatal outcomes between long-term and short-term use of tocolytic agent: a historical cohort study in a single perinatal hospital. J Obstet Gynaecol Res. 2016; 42:16801685.

10. European Medicines Agency. PRAC recommends restricted use of short-acting beta-agonists in obstetric indications. https://www.ema.europa.eu/en/documents/referral/shortacting-beta-agonists-article-31-referral-prac-recommendsrestricted-use-short-acting-beta_en.pdf (accessed January 14, 2021).

11. Actual usage of ritodrine preparations in Japan (KISSEI PHARMACEUTICAL CO., LTD. In-house materials) 2014. (in Japanese)

12. UTEMERIN Tab $5 \mathrm{mg}$. Package insert. KISSEI PHARMACEUTICAL CO., LTD.; 2016. (in Japanese)

13. UTEMERIN injection $50 \mathrm{mg}$. Package insert. KISSEI PHARMACEUTICAL CO., LTD.; 2015. (in Japanese)

14. Cotton DB, Strassner HT, Lipson LG, Goldstein DA. The effects of terbutaline on acid base, serum electrolytes, and glucose homeostasis during the management of preterm labor. Am J Obstet Gynecol. 1981; 141:617-624.

15. Brazy JE, Little V, Grimm J. Isoxsuprine in the perinatal period. II. Relationships between neonatal symptoms, drug exposure, and drug concentration at the time of birth. J Pediatr. 1981; 98:146-151.

16. UTEMERIN Tab $5 \mathrm{mg}$. Interview Form. KISSEI PHARMACEUTICAL CO., LTD.; 2016. (in Japanese)

17. UTEMERIN injection $50 \mathrm{mg}$. Interview Form. KISSEI PHARMACEUTICAL CO., LTD.; 2015. (in Japanese)

Received February 10, 2021; Revised February 19, 2021; Accepted February 23, 2021.

*Address correspondence to:

Yuriko Yonaga, Department of Pharmaceutical Sciences, Teikyo Heisei University, 4-21-2 Nakano, Nakano-ku, Tokyo, 164-8530, Japan.

E-mail: y.yonaga@thu.ac.jp

Released online in J-STAGE as advance publication February 27, 2021. 